\section{s. Kohane}

arvard Medical School,

hildren's Hospital Informatics Program, Medical School and Children's Hospital, trision of Endocrinology,

children's Hospital,

Boston, USA

In 1970, Dr. William Schwartzboldly predicted that "it seems probable that in the not too distant future the physician and the computer will engage in frequent dialogue, the computer continuously taking note of history, physical findings, laboratory data, and the likes" [1]. In 1991, the Institute of Medicine reported [2] "No operational clinical information system in 1990 can manage the entire patient record with all its inherent complexities". In this "perspective, any prediction for future success appears risky, yet the dissatisfaction with the medical record appears to precede its electronic instantiation. Over one hundred years ago, Florence Nightingale wrote, "in attempting to arrive at the truth, I have applied everywhere for information, but in scarcely an instance have I been able to obtain hospital records fit for any purposes of comparison. If they could be obtained... they would show subscribers how their money was being spent, what amount of good was teally being done with it, or whether the money was doing mischief rather than good..."

Part of the problem may be the multiplicity of goals that various parties Wish to place on the fragile shoulders of medical record systems, paper or fectronic [3]. To enumerate only a êw of these goals: communication between care providers, medico-legal

\title{
Synopsis
}

\section{Computer-based Patient Records}

protection, outcomes analysis, longitudinal record for care, medical discovery, quality control and real-time decision support. Efficacy in meeting one of the goals does not at all predict efficacy in meeting another goal, particularly in the implementation of Computer-based Patient Records (CPR's). For example, it may be that for the goal of communication between providers, unstructured narrative text viewed linearly or via a fast, indexed, text search engine might be adequate. For outcomes analysis however, such a record without any controlled vocabulary or structure is highly inadequate. Furthermore, the design, engineering and sociology of deploying a structured record with a controlled vocabulary is quite different than that of a narrative, unstructured record. Therefore, it is quite predictable that even if a system were to be implemented that served one of the CPR goals very well, there would be substantial criticism regarding its failure to meet the other CPR goals. Yet, even a simple unstructured CPR geared for communication, would already represent a much higher degree of function than the current, paper-based patient record in its accessibility, shareability, searchability and compact archival form.

The aforementioned problem notwithstanding, informaticians, at least those with any longevity in this field, are apparently a group of irrepressible optimists. Despite the difficulty in meeting the multitudinous goals of the CPR, efforts to achieve the holy grail, the allpurpose CPR, have continued for well over thirty years. Part of this optimism may be justified by the accumulation of wisdom and the never-ending progress of ever more leveraged new information technologies. The worry however, is that some of the optimism stems from a lack of knowledge of the many ways CPR efforts in the past have succeeded or failed and we will be doomed to revisit our errors generation after generation. In this light, the review by Tange et al [4] is particularly valuable. They have provided a chronologically organized description of the development of CPR's, carefully highlighting the contributions made by each CPR but also tactfully delineating the ways in which these systems have not succeeded or fully met their designers' expectations. To provide the reader with an overarching framework, the review is partitioned across two dimensions. The first dimension distinguishes "classical" systems from the "experimental" systems. The second dimension is defined by three major themes: data entry, user interface, and machineability of data. The review explicitly omits evaluating all current commercially available systems. This is understandable but unfortunate as an impartial review of commercial 
CPR's within the framework provided by Tange et al. [4] would be of great utility. Regarding the non-commercial systems, they make quite a few interesting, if controversial points. First they note that the classical systems, developed in the 70's and 80's, rarely have much in the way of clinician-entered data and rather focus on integrating non-clinician-derived data (e.g. laboratory results, administrative data). Furthermore, they note that these systems have been difficult to adapt to modern user interfaces, and data entry techniques. With the advent of "webwrapping" of legacy systems, their conclusion may be correct at this time, but premature. The experimental systems, in contrast, provide several intriguing examples of methods to enable clinicians to enter data. These methods range from full control of clinician entry through intelligent user interfaces to post-processing of narrative with natural language techniques. The reviewers point out that in all evaluations to date, for the purpose of clinical care, clinicians prefer a natural language summary, whether it is generated de novo from data coded in a controlled vocabulary or represents the original prose of the clinician. Unfortunately, the reviewers also make it clear that none of these techniques has been so successful as to dominate the others, nor have very many of the experimental systems made the transition to largescale clinical use.

Nguyen et al. [5] report on a case history of "experimental systems" (in the sense of Tange et al. [4]) that have made the transition to full clinical use successfully. They describe a study in which they evaluated the performance of a narrative report generator they call "Professor Belmonte" which has been used since June of 1996. The particular application domain is bone marrow aspirates and the reports generated, from struc- tured data elements, are narrative summaries. These summaries not only abstract the findings, but also provide an interpretation of histology and differential diagnosis. Overall, domain experts in this retrospective study rated the automatically generated reports as of comparable quality to the human generated ones. Although this study does not speak to the problem of data entry, it does demonstrate that once captured, structured data in some domains can be effectively used to generate acceptable narrative reports.

Once we do have successful fullfledged CPR's, it is highly likely that we will wish to share data among them. The history of the database technology behind CPR's has made data sharing efforts quite challenging. One important reason is that, until recently, few CPR's were built upon relational databases and could not therefore exploit modern database design techniques. Pierik et al. [6] follow the path set by Johnson [7] in the application of rational relational database design methodology to the task of building shared or re-usable data repositories for CPR's. In addition to the judicious use of normalization, the creation of a core set of generic, time-stamped relations was identified as essential for the long-term evolution and maintainability of the multi-purpose database. Resolution of semantic ambiguity or incompleteness were also identified as important tasks. However, experience with truly disparate databases suggests that such resolution is very difficult if at all possible [8]. Nevertheless, as the next generation of "classical" CPR's are implemented, the techniques described by Pierik et al. [6] and Johnson [7] will prevent many of the most common errors in CPR database implementation.

Anne Birgitte Als [9] describes a cautionary study that provides sot hints as to how the presence of a can have a negative effect on patient-clinician relationship. Usi well-established sociological tec niques, she studied clinicians' intera tions with patients in the presence desktop computers, which have be come quite prevalent in Denmark [10

These interactions, documented videotape, were occasionally quite stri ing, particularly when the compute would be referred to as if it was a oracular "Magic Box". For example "a mother brought her 2-year-old chile for a check-up after pneumonia. The GP examines the child, then looked fo a while at his screen, pointed to it, an said he is getting better, no doubt of that". Even more frequently, the computer was used as a prop by the clinis cian to allow for interruption of the conversation with the patient. Wher interviewed afterwards, most of the clinicians were surprised and uncom. fortable with the way the compute seemed to intrude in their conversation. Similarly, patients were often mystified or annoyed by the interposition of the computer in the office, and also worried about the threat that it posed to their privacy. Other patients. did believe that the computer helped their physician remain current and competent. Perhaps the most important lesson to be drawn from the study is that when computers become part of the clinician-patient conversation, itis important at the outset for clinicians 10 understand how it is changing their style of practice and to explain to their patients, the computer's presence and use. It also does suggest that wherr CPR's truly become ubiquitous the effect on medical care will likely be larger and different than we have expected. Perhaps, the best we can hope for is that if Dr. Schwartz's predictions are realized, the dialogue between physician and computer will always defer to the patient-physician conversation 
rerences

SchwartzWB. Medicine and the computer: ceprontise and problems of change. New o. J Med 1970;283:1257-64. thte of Medicine. The Computer-based Patient Record: An Essential Technology PrHealth Care. Washington DC: National Academy Press; 1997 (revised version).

3. Van Ginneken AM, Stam H, Moorman PW.A multi-strategy approach for medical Tords of specialists. Int J Biomed Comput 1996;42:21-6.

4. Tange HJ, Hasman A, De Vries Robbé PF, Schouten HC. Medical narratives in electronic medical records. Int J Med Informatics 1997;46:7-29.

5. Nguyen DT, Diamond LW, Cavenagh JD, Parameswaran R, Amess JA. Haematological validation of a computerbased bone marrow reporting system. J Clin Pathol 1997;50:375-8.
6. Pierik FH, Van Ginneken AM, Timmers T, Stam H, Weber RF. Restructuring routinely collected patient data: ORCA applied to andrology. Meth Inform Med 1997;36:184-90.

7. Johnson SB. Generic data modeling for clinical repositories. J Am Med Inform Assoc 1996;3:328-39.

8. Kohane IS, Van Wingerde FJ, Fackler J, Safran C, Rind D, Kilbridge P, et al. Sharing electronic medical records across multiple heterogeneous and competing institutions. In: Cimino JJ, ed.Proceedings of the 1996 AMIA Fall Symposium. Philadelphia: Hanley \& Belfus Inc, 1996:608-12.

9. Als AB. The desk-top computer as a magic box: patterns of behaviour connected with the desk-top computer; GPs' and patients' perceptions. Fam Pract 1997;14:17-23.

10. Stroppe J, Maeseneer JD, Ceenaeme R. A picture of primary health care in Europe. In: Maeseneer JD, Beolchi L, eds. Telematics in Primary Care. Amsterdam: IOS Press, 1995:1-30.

Address of the author:

Isaac $\mathrm{S}$. Kohane, $\mathrm{MD}, \mathrm{PhD}$,

Assistant Professor, Harvard Medical

School, Director, Children's Hospital

Informatics Program,

Medical School and Children's Hospital,

Division of Endocrinology,

224 Wolbach,

Children's Hospital,

300 Longwood Avenue,

Hunnewell 3rd Floor

Boston, MA 02115,

USA

e-mail:gasp@medg.lcs.mit.edu and

kohane@a1.tch.harvard.edu 